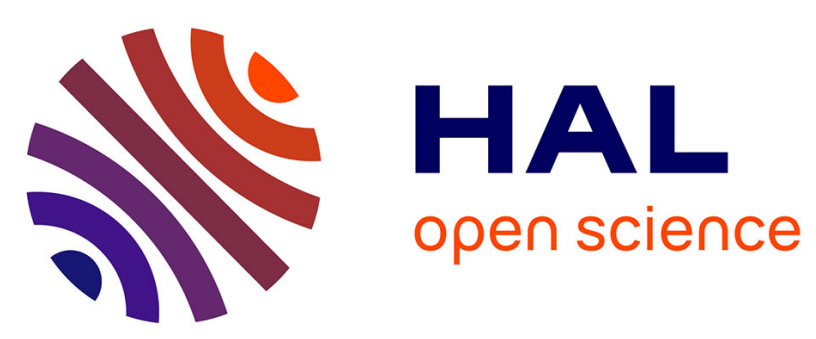

\title{
Crystalline orientation assessment in transversely isotropic semicrystalline polymer: Application to oedometric compaction of PTFE
}

Gabriel Guenoun, Nicolas Schmitt, Stéphane Roux, Gilles Régnier

\section{To cite this version:}

Gabriel Guenoun, Nicolas Schmitt, Stéphane Roux, Gilles Régnier. Crystalline orientation assessment in transversely isotropic semicrystalline polymer: Application to oedometric compaction of PTFE. Polymer Engineering and Science, 2021, 61 (1), pp.107-114. 10.1002/pen.25561 . hal-03158759

\author{
HAL Id: hal-03158759 \\ https://hal.science/hal-03158759
}

Submitted on 4 Mar 2021

HAL is a multi-disciplinary open access archive for the deposit and dissemination of scientific research documents, whether they are published or not. The documents may come from teaching and research institutions in France or abroad, or from public or private research centers.
L'archive ouverte pluridisciplinaire $\mathbf{H A L}$, est destinée au dépôt et à la diffusion de documents scientifiques de niveau recherche, publiés ou non, émanant des établissements d'enseignement et de recherche français ou étrangers, des laboratoires publics ou privés. 


\title{
Crystalline orientation assessment in transversely isotropic semicrystalline polymer: Application to oedometric compaction of PTFE
}

\author{
Gabriel Guenoun $^{1,2,3}$ (1) | Nicolas Schmitt ${ }^{1,4}$ | Stéphane Roux ${ }^{1}$ ｜ Gilles Régnier
}

${ }^{1}$ LMT, ENS-Paris-Saclay/CNRS, Université Paris-Saclay, Cachan, France ${ }^{2}$ PIMM, Arts et Métiers, CNRS, CNAM, HESAM Université, Paris, France

${ }^{3}$ Saint-Gobain Research Paris, Aubervilliers, France

${ }^{4}$ INSPE, Université Paris-Est Créteil Valde-Marne, Saint-Denis, France

Correspondence

Gabriel Guenoun, PIMM, Arts et Métiers, CNRS, CNAM, HESAM Université, 151 boulevard de l'Hôpital, Paris 75013, France.

Email: gabriel.guenoun@saint-

gobain.com

Funding information

CIFRE PhD

\begin{abstract}
The Hermans orientation factor is a scalar giving a simple assessment of molecular or crystal orientation in a polymer. It is usually determined from X-ray diffraction measurements. The evaluation of the Hermans orientation factor is proposed from the diffraction analysis of only a single diffracting plane. The estimation relies on (a) the assumption that the polymer crystals possess a cylindrical symmetry with respect to the chain direction and (b) that the processing conditions induce a transverse isotropy of the polymer chains. A practical consequence of the proposed analysis is that the Hermans orientation factor can be computed from a single 1D diffractogram. This enables fast evaluations since even low signal-to-noise ratio signals can be processed with optimal noise reduction. An illustration of this method for uniaxially compacted polytetrafluoroethylene (PTFE) powder is presented. The crystalline orientation is followed during in situ crystallization experiments with a fine time resolution.
\end{abstract}

\section{KEYWOR D S}

crystalline orientation, crystallization, PTFE, transverse isotropy, X-ray diffraction

\section{1 | INTRODUCTION}

The characterization of the molecular orientation in a polymer is of prime importance when evaluating its properties, and especially those related to mechanical behavior. ${ }^{[1]}$ This orientation results mainly from processing conditions $^{[2]}$ and thus measuring it is essential to optimize the manufacturing in view of target applications.

The characterization of molecular orientation has been described in detail elsewhere ${ }^{[3]}$ in reference to an orthogonal coordinate axis system attached to the sample, typically the Machine-Transverse-Normal (M-T-N) system. An orthogonal Cartesian coordinate axis system $x-y-c$ is defined for a polymer chain with the $c$ direction generally defined as the polymer chain direction, $x$ and $y$ directions are defined in order to characterize the orientation of the considered molecular structure around $c$ axis. The orientation of a macromolecular chain frame can be defined by the three Euler angles $\alpha, \varphi, \psi$ in the M$\mathrm{T}-\mathrm{N}$ axis system. The probability of finding a molecule chain at the specific orientation $(\alpha, \varphi, \psi)$ can be expressed by the following orientation distribution function (ODF) as a sum of the generalized Legendre functions $\left[Z_{l}(\cos \alpha)\right]$,

$$
Y(\alpha, \varphi, \psi)=\sum_{l=0}^{\infty} \sum_{m=-l n=-l}^{+l} \sum_{l m n}^{+l} Z_{l}(\cos \alpha) e^{-i m \varphi} e^{-i n \psi}
$$

where $P_{\operatorname{lmn}}$ are coefficients to be determined. As materials have generally an orthorhombic symmetry, only $P_{\operatorname{lmn}}$ with even indices $l, m$, and $n$ are nonzero. 
If a cylindrical symmetry with respect to the polymer chain holds, or in other words, if there is no preferential orientation around axis c, which is often the case for polymers, $\psi$ is irrelevant, and hence all $P_{\operatorname{lmn}}$ for $n \neq 0$ are null. Only second-order moments are often considered to assess the molecular orientation distribution in most studies as it usually contains more information than other higher orders. Hence, it is quite complex to determine other moments to get more precision. Experimental techniques such as infrared dichroism allow determining only these second-order moments. ${ }^{[4]}$ Thus, if the molecular orientation can be considered as uniaxial, only one of the second-order moments remains: $P_{200}$, which corresponds to the Hermans orientation factor $f_{c}:{ }^{[5,6]}$

$$
f_{c}=\frac{1}{2}\left(3\left\langle\cos ^{2} \alpha\right\rangle-1\right)
$$

The Hermans orientation factor is a scalar descriptor, which quantifies in that case the chain orientation with respect to the Machine direction. This factor is null when the distribution is isotropic and ranges from -0.5 , when all the chains are perpendicular to the reference direction, to 1 when all the chains are aligned with it. Even though a Hermans orientation factor does not capture the complete orientation information as it is only the first relevant term in the moment series expansion (Equation (1)), it reveals very convenient to compare different textures as they are condensed to a scalar which is in fact the most relevant one, even if it does not characterize uniquely the probability distribution function of orientations. Extra refinements can be obtained from the fourth-order moments ${ }^{[7]}$ measured through Raman spectroscopy or wide-angle X-ray scattering (WAXS).

WAXS is one of the experimental techniques, which offers the most complete tools to assess molecular orientation and especially crystalline orientation. The diffracted intensity in a given direction can be related to the amount of crystals that meets Bragg's condition. Scanning this diffracted intensity in different directions gives information on the distribution of orientation of the crystalline planes. Therefore, sweeping all space directions for a given Bragg's condition completely defines the ODF of diffracted planes with respect to a sample axis system and allows building pole figures. ${ }^{[8]}$ This ODF of diffracted plane normals can be described by the same ODF formalism as that of molecular orientation (Equation (1)) with only two angles $Y(\alpha, \varphi)$ as the rotation about the normal is irrelevant. A Hermans orientation factor of the $h \mathrm{kl}$ diffracted plane can be calculated to evaluate its firstorder orientation terms. The mean cosine square of an $h k l$-diffracted plane intensity in reference to sample coordinate axis system can be calculated as: ${ }^{[3]}$

$$
\begin{aligned}
\left\langle\cos ^{2} \alpha_{h k l, M}\right\rangle & =\frac{\int_{0}^{2 \pi} \int_{0}^{\pi / 2} I(\alpha, \varphi) \cos ^{2} \alpha \sin \alpha d \alpha d \varphi}{\int_{0}^{2 \pi} \int_{0}^{\pi / 2} I(\alpha, \varphi) \sin \alpha d \alpha d \varphi}=\left\langle\cos ^{2} \alpha\right\rangle \\
\left\langle\cos ^{2} \alpha_{h k l, T}\right\rangle & =\left\langle\sin ^{2} \alpha \cos ^{2} \varphi\right\rangle \\
\left\langle\cos ^{2} \alpha_{h k l, N}\right\rangle & =\left\langle\sin ^{2} \alpha \sin ^{2} \varphi\right\rangle
\end{aligned}
$$

The same methodology can be applied to determine the orientation of amorphous phase by WAXS analysis considering the intensity of the amorphous halo. ${ }^{[9]}$ However, the quantification of macromolecular chain orientation, which is also directly linked to mechanical properties, usually requires the diffraction measurements of several crystal planes because one such, normal to the chain, is either not available, or its diffraction angle is too large to be accessed, or its peak intensity too small to be exploited accurately. For polyethylene terephthalate, an approximation is often done by using the diffracted plane $(105)^{[10]}$ because its normal is close to the chain direction, but this approximation does not hold for most of semicrystalline polymers.

From the initial work of Stein on polyethylene (PE) ${ }^{[11,12]}$ Wilchinski, ${ }^{[13]}$ and then Sack ${ }^{[14]}$ theorized the equations linking diffraction measurements of several crystalline planes with macromolecular chain orientation. In the case where the analyzed diffraction planes contain the chain axis (c axis), two sets of planes are sufficient. For example, crystalline orientation was characterized in the case of polypropylene (PP) using diffraction planes (040) and (110): ${ }^{[15,16]}$

$$
\left\langle\cos ^{2} \alpha_{c, J}\right\rangle=1-1.1\left\langle\cos ^{2} \alpha_{110, J}\right\rangle-0.9\left\langle\cos ^{2} \alpha_{040, J}\right\rangle
$$

where $J$ is a sample reference direction. For polyvinylidene fluoride (PVDF) (110) and (020) planes are used $^{[17]}$ while for polyethylene (PE) exploited planes are (110) and (200). ${ }^{[18-21]}$

In this article, it will be shown that assuming (a) that the polymer crystals possess a cylindrical symmetry with respect to the chain direction and (b) that the processing conditions induce a transverse isotropy of the polymer chains in the Machine direction, one can evaluate the Hermans chain orientation factor with respect to the Machine direction with no more than a single diffraction plane scan containing the chain axis. This technique is further applied to measurements performed on an oedometrically compacted polytetrafluoroethylene (PTFE) powder in which both of these conditions are 
met. In addition, high signal-to-noise ratio signals can be processed enabling fast analyses of in situ acquisitions during crystallization.

\section{2 | METHODOLOGY DEVELOPMENT}

\section{1 | Geometrical modeling of crystalline orientation}

Our development is based on Wilchinky's work ${ }^{[13]}$ summarized here to help understanding the development. Let us consider the sample axis system Machine-TransverseNormal of a semicrystalline polymer (Figure 1). The angle between a crystallographic axis $c$, which (in most cases) is the macromolecular chain axis, and the machine direction is noted $\sigma$. The crystallographic axis $c$ coincides with the $z$ axis of an orthogonal Cartesian coordinate system $x-y-z$, such that $x$ and $y$ axes define the orientation of the crystal. Let us note that these axes $x$ and $y$ are not generally the crystallographic axes noted classically $a$ and $b$, because the axis system $a-b-c$ only forms an orthogonal axis in the case of an orthorhombic crystalline cell. The normal to a diffracting plane $(h k l)$ is defined as a unit vector in the sample axis system M-T-N by two Euler angles $\alpha$ and $\varphi$, and its coordinate in $x-y$-c coordinate system are:

$$
\overrightarrow{n_{h k l}}=e \vec{x}+f \vec{y}+g \vec{z}
$$

where $e, f$, and $g$ are the direction cosines with respect to $x, y$, and $z$ respectively and result from the crystal orientation.

$$
\begin{aligned}
& \text { As } n_{h k l} \cdot M=\cos \theta, \text { it can be shown that: }{ }^{[13]} \\
& \begin{aligned}
\left\langle\cos ^{2} \alpha\right\rangle= & e^{2}\left\langle\cos ^{2} \delta\right\rangle+f^{2}\left\langle\cos ^{2} \varepsilon\right\rangle+g^{2}\left\langle\cos ^{2} \sigma\right\rangle \\
& +2 e f\langle\langle\cos \delta\rangle\langle\cos \varepsilon\rangle\rangle+2 f g\langle\langle\cos \sigma\rangle\langle\cos \varepsilon\rangle\rangle \\
& +2 e g\langle\langle\cos \sigma\rangle\langle\cos \delta\rangle\rangle
\end{aligned}
\end{aligned}
$$

As $x-y-z$ is an orthogonal Cartesian axis system, the orthogonality property results in:

$$
\left\langle\cos ^{2} \delta\right\rangle+\left\langle\cos ^{2} \varepsilon\right\rangle+\left\langle\cos ^{2} \sigma\right\rangle=1
$$

To determine $\left\langle\cos ^{2} \sigma\right\rangle$, affinely related to the Hermans crystalline factor with respect to the machine direction, five unknowns remain among the six average cosines. The orientation measurements of five diffracting planes having normals in different directions

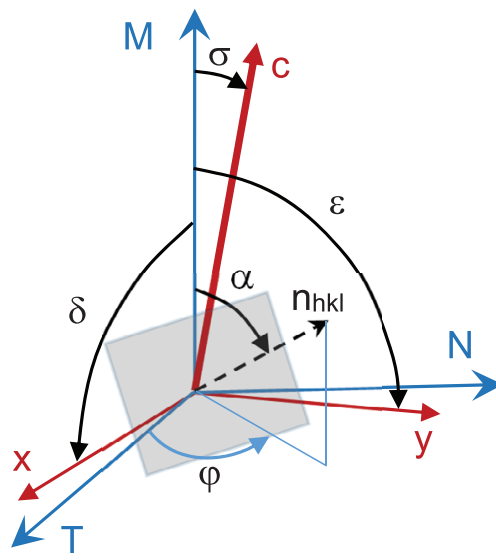

FI G URE 1 Angles definition of the crystalline Cartesian coordinate system $\mathrm{x}-\mathrm{y}-\mathrm{c}$ (with $\mathrm{c}$ the chain direction) and of a diffracting plane (defined by its normal $n_{\mathrm{hkl}}$ ) with respect to $\mathrm{M}$ in the sample axis system M-T-N [Color figure can be viewed at wileyonlinelibrary.com]

would provide the five necessary equations. In practice, the number of equations might be reduced considerably. If a $(h k 0)$ plane is considered, only two unknowns are left in Equation (6) since $g=0$. Then the orientation distribution measurement of two $(h k 0)$ planes is necessary to determine the Hermans orientation factor of molecular chains. ${ }^{[13-17]}$ All these developments can be repeated for another crystallographic axis with respect to sample axis $\mathrm{T}$ or $\mathrm{N}$.

If the orientation is not uniaxial, it is worth noting that the only determination of the Hermans orientation factor of a crystallographic axis with respect to machine direction gives only a very poor representation of the ODF. For an orthotropic orientation or biaxial orientation like in the one induced in injection molding, three Hermans orientation factors give a better assessment ${ }^{[7]}$, although one of them can be deduced from an equation similar to Equation (7), namely

$$
f_{c, M}+f_{c, T}+f_{c, N}=0
$$

\section{2 | Diffracting plane containing the chain with cylindrical symmetry with respect to polymer chain}

Diffracting planes containing the crystal chains are often accessible with a high diffraction intensity and an easier possible deconvolution. For example, the plane (110) in the case of PE, PP, or PVDF or the plane (100) for PTFE is easily accessible for diffraction measurements. For PTFE, the plane (llll $\left.\begin{array}{lll}0 & 0 & 15\end{array}\right)$ would allow assessing chain 
orientation; ${ }^{[22-24]}$ however, this peak has a very small intensity, and diffracts at high angle $\Theta / 2 \Theta\left(\sim 72^{\circ}\right.$ for $\mathrm{CuK} \alpha$ radiation) and requires a deconvolution with amorphous halo and another peak, inducing a lot a dispersion in the results. Some authors suggest considering an orientation factor calculated from (100) plane without doing a quantitative link with chain orientation. ${ }^{[25]}$

Consider orthorhombic, hexagonal or pseudohexagonal crystal class in which the crystallographic axes $a$ and $b$ are perpendicular the chain axis $c$. For these crystal classes, we assume that there is a cylindrical symmetry with respect to the polymer chains, in other words, that only the chain axis orientation is important without any preferential orientation of crystallographic axes $a$ and $b$ with respect to chain axis. This assumption, which is often found to hold for polymers, especially for hexagonal crystal structure, leads to

$$
f_{a, J}=f_{b, J}
$$

where $J$ is a sample direction axis $\mathrm{M}$, T, or $\mathrm{N}$.

In the case of an orthorhombic crystal, $a$ and $b$ are equivalent to $x$ and $y$ in the previously defined $x-y-c$ coordinate system. Therefore, the orthogonality relationship in Equation (7) gives a relationship between Hermans factors of crystallographic axes:

$$
f_{a, J}+f_{b, J}+f_{c, J}=0
$$

It is noteworthy that this relationship is actually still valid in the case of a hexagonal or pseudo hexagonal crystal as $a$ and $b$ play an equivalent role as $x$ and $y$ due to the cylindrical symmetry (Equation (9)).

In some other cases where this assumption is not guaranteed, it is necessary to validate it in the first place with an extra WAXS experiment.

Therefore, according with the cylindrical symmetry hypothesis, the Hermans factor of chain orientation $f_{c}$ can be determined as:

$$
f_{c, J}=-2 f_{a, J}
$$

Measuring the crystal chain orientation is therefore equivalent to measuring the orientation of its normal, which can be accessed via a diffracting plane containing the chain.

\section{3 | Transversely isotropic material}

Furthermore, the material can be transversely isotropic due to processing conditions. This is the case for oedometric compaction of PTFE powder. The transverse isotropy simplifies further the diffraction measurement as it is no longer mandatory to access the full pole figure, but only a $2 \mathrm{D}$ measurement. Instead of recording the diffracted intensity $I(\alpha, \varphi)$ for both arguments, only the $\alpha$ dependence is necessary as $I$ is invariant with respect to $\varphi$. Then Equation (1) becomes

$$
\left\langle\cos ^{2} \alpha_{a, M}\right\rangle=\frac{\int_{0}^{\pi / 2} I\left(\alpha, \varphi_{0}\right) \cos ^{2} \alpha \sin \alpha d \alpha}{\int_{0}^{\pi / 2} I\left(\alpha, \varphi_{0}\right) \sin \alpha d \alpha}
$$

where $I$ is obtained for only one configuration of $\varphi=\varphi_{0}$.

Hence the Hermans orientation factors for $a$ or any crystallographic axes can be computed relatively simply according to Equation (12). This provides a very noiserobust evaluation, which, in turn, is compatible with a fine temporal resolution for which very low signal-tonoise ratios are expected in the diffractogram. This is particularly beneficial when the measurement is done with a one-dimensional sensor scanning along $\alpha$.

A mere quadrature of the integral shown in Equation (12) can be performed at accordingly selected positions of $\alpha$ in order to reduce the measurement uncertainty. This is particularly true when the X-ray diffraction is acquired with a one dimensional sensor through scanning along $\alpha$.

Transverse isotropy is a very common feature in polymer science. Combined with the statistical transverse isotropy assumption, very convenient crystalline orientation characterization can be achieved thanks to X-ray diffraction experiments. An experimental setup suited to perform such measurements is illustrated in the next section in the case of uniaxially compacted PTFE.

\section{3 | ORIENTATION MEASUREMENTS ON COMPACTED POWDER PTFE}

\section{1 | Material}

PTFE cubes were obtained from cold oedometric compaction of a fine PTFE powder (between 20 and $200 \mu \mathrm{m}$ ) having an ultra-high molecular mass. The process was performed at ambient temperature under a pressure of $50 \mathrm{MPa}$ and maintained in the same geometry for $100 \mathrm{~min}$. A die is filled with powder and pressure is applied uniaxially (Figure 2(A)). The obtained sample is therefore transversely isotropic due to the axial symmetry of the process and the powder isotropy. Note that skin effects could modify this symmetry but in practice due to 

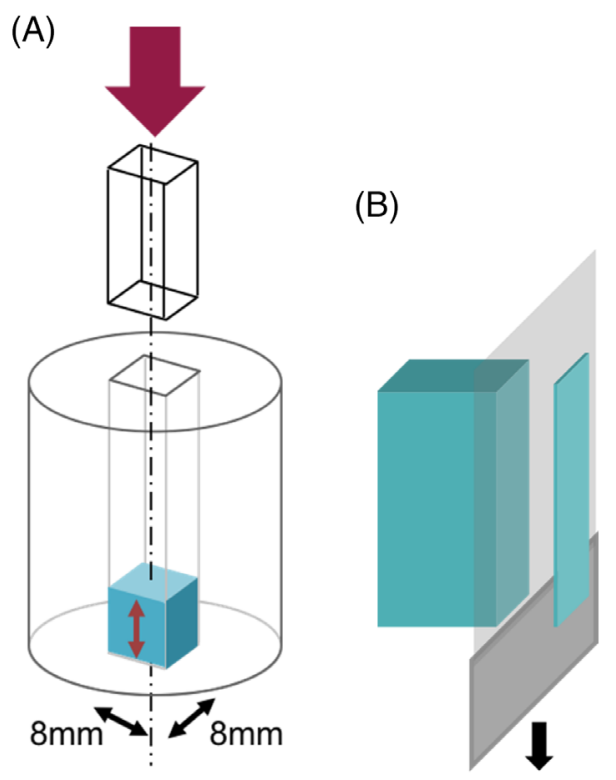

FI G URE 2 (A) PTFE powder is compacted uniaxially in a die. (B) The compacted block is then cut using a microtome to obtain $300 \mu \mathrm{m}$ thick PTFE slices [Color figure can be viewed at wileyonlinelibrary.com]

PTFE low coefficient of friction, it is safe to assume that transverse isotropy holds in the bulk material away from the specimen skin that may be affected.

Slices of PTFE are obtained from the PTFE blocks using a microtome (Figure 2(B)). They are about $300 \mu \mathrm{m}$ thick for the lab experiment and $100 \mu \mathrm{m}$ thick for the experiment in the synchrotron facility. The reduction of thickness is linked to the heating plate used to restrain the thermal gradient. Those slices are such that they contain the compaction direction (CD) and one transverse direction (TD).

\section{2 | Orientations for green and sintered PTFE}

Two samples were studied, one slice of green PTFE compact and one slice of sintered PTFE. Sintering means PTFE was melted and then recrystallized. The objective was to determine whether sintering could modify the crystalline texture induced by uniaxial compaction.

Both samples were tested using a Philips X'Pert X-ray diffractometer with a copper source (with a generator voltage of $45 \mathrm{kV}$ and a tube current of $40 \mathrm{~mA}$ ). X-ray diffraction was performed in transmission with a $\theta-\theta$ configuration (Bragg's condition) as shown in Figure 3. An azimuthal scan was done by varying $\alpha$ over $180^{\circ}$ at $2 \theta=18.3^{\circ}$ corresponding to PTFE diffraction peak (100)

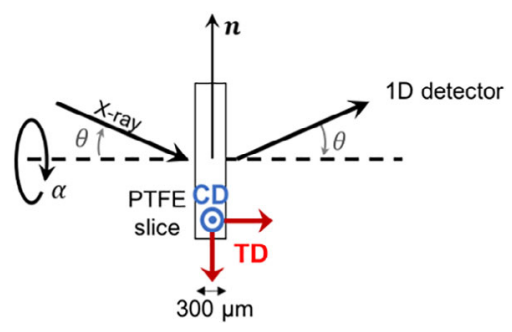

FIG URE 3 Sketch of the $\theta-\theta$ diffraction setup. The sample is rotated along the horizontal axis to record the diffracted intensity along $\alpha$. The normal $n$ is rotating in the PTFE slice from the compaction direction (CD) for $\alpha=0^{\circ}$ to the transverse direction (TD) for $\alpha=90^{\circ}$ [Color figure can be viewed at wileyonlinelibrary.com]

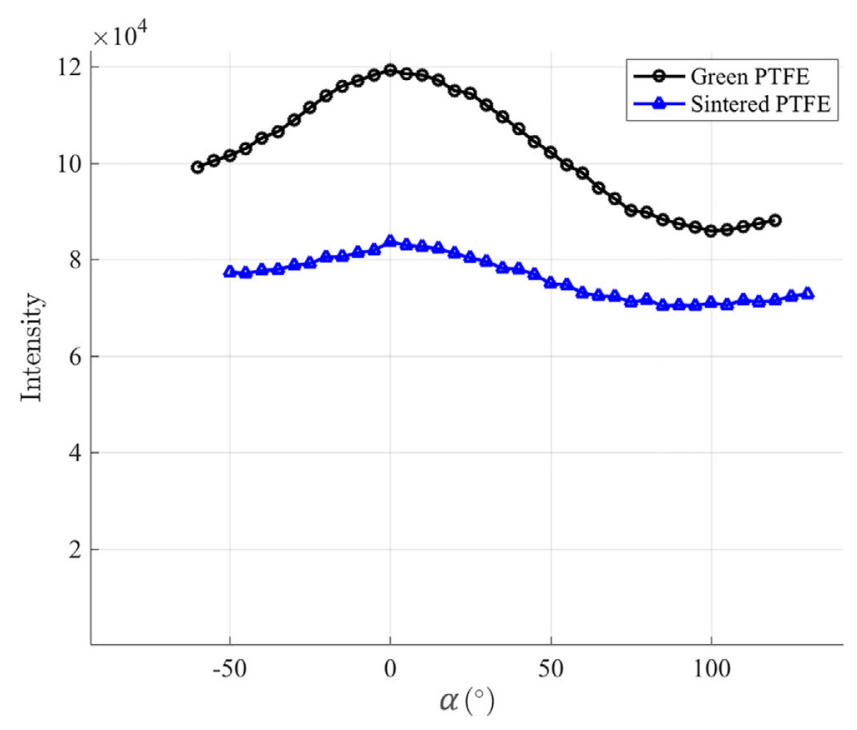

F I G U RE 4 Integrated diffracted signal along peak (100) as function of azimuthal angle $\alpha$ for a green PTFE sample and for a sintered PTFE sample [Color figure can be viewed at wileyonlinelibrary.com]

for $\mathrm{K} \alpha \mathrm{Cu}$ radiation. One experimental value was obtained for each $\varphi$ position. The position $\alpha=0^{\circ}$ was chosen to correspond to $\mathrm{CD}$ and $\alpha=90^{\circ}$ to TD.

Experimental data sets obtained for both samples are presented in Figure 4. The two signals reveal anisotropic crystalline textures as the intensity is higher in CD than in TD. The sintered PTFE seems to have a lower anisotropy than green PTFE.

By using the method described above, the Hermans factor $f c$ (Equation (2)) can be evaluated for both samples. For green PTFE, $f c=-0.083$, and for sintered PTFE, $f c=-0.043$. It means that the orientation of the PTFE crystals is as expected mostly perpendicular to the $\alpha=0$ direction chosen to be the oedometric CD. However, the level of orientation is quite far from a complete 


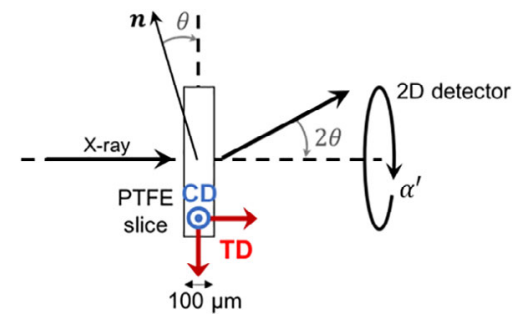

F I G URE 5 Sketch of the diffraction setup allowing to get a 2D signal per capture. A drawback is that the diffraction normal is no more included in the plane of the PTFE slice. CD, compaction direction; TD, transverse direction [Color figure can be viewed at wileyonlinelibrary.com]

orientation in the transverse plane $(f c=-0.5)$. It also shows that sintering reduces the crystalline texture, which could be partly explained by stress relaxation. ${ }^{[26]}$ The melting stage might allow the macromolecules to relax and somehow to reduce the preferential orientation induced by compaction.

Studying the evolution of the crystalline texture at crystallization could provide insights on the different mechanisms. Evaluating the Hermans orientation factor with the method described herein allows one to measure the crystalline orientation in in situ experiments.

\section{3 | Evolution of orientation during in situ crystallization}

The ESRF synchrotron facility offered the opportunity to perform experiments on the D2AM line. Wide angle Xray scattering experiments were carried out on $100-\mu \mathrm{m}$ thick PTFE slices. The latter were placed on a Linkam heating stage THMS600 that can reach $150^{\circ} \mathrm{C} / \mathrm{min}$ of heating/cooling rate. The thickness of the PTFE slice is reduced compared to previous experiments to limit the thermal gradient produced by the heating stage. PTFE diffractograms were captured all along the crystallization thanks to the high intensity and high resolution of the beamline. Analyzing the crystalline peak during crystallization gives access to the evolution of the crystallinity. It can then be compared to measurements made with differential scanning calorimetry. Similar in situ experiments have been performed on other polymers to study the microstructural evolution during thermal treatment. $^{[27]}$

Another interesting piece of information provided by the in situ crystallization is the evolution of the crystalline orientation. The PTFE crystallization can be decomposed into primary and secondary crystallization mechanisms (as it is the case for many semicrystalline polymers). ${ }^{[28-30]}$ The study of the crystalline texture gives

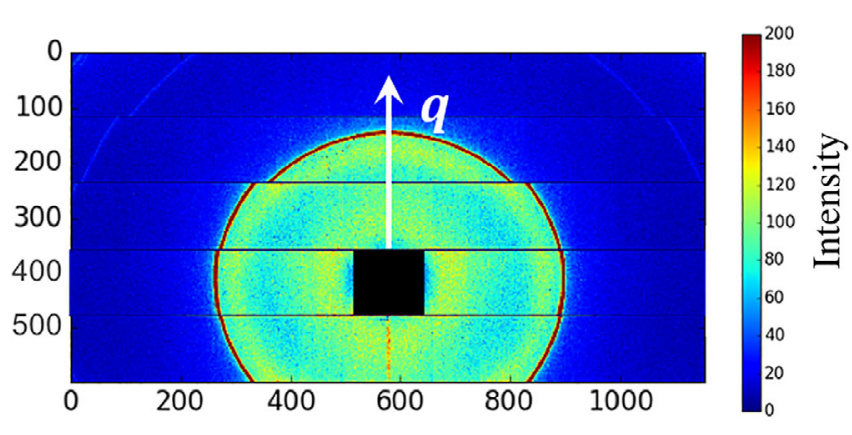

F I G U R E 6 2D diffraction pattern realized at ESRF synchrotron facility. The diffraction circles are slightly shifted as the capture is performed via 10 spaced-apart detectors strips [Color figure can be viewed at wileyonlinelibrary.com]

the opportunity to determine if both mechanisms induce the same orientation or not. Such information is essential to better understand the microstructural mechanisms responsible for the different crystallization processes and how they are related.

Figure 5 shows the diffraction setup used for the in situ measurements on D2AM BM02 line in ESRF synchrotron. The $\mathrm{X}$-ray beam $(8 \mathrm{KeV})$ is perpendicular to the PTFE sample and the diffracted X-rays are collected with a $2 \theta$ angle on the $2 \mathrm{D}$ detector placed $10 \mathrm{~cm}$ from the sample. The sample was exposed $1 \mathrm{~s}$ at an acquisition frequency that was adjusted between 0.1 and $0.5 \mathrm{~Hz}$ depending on the proximity to the crystallization temperature. Dark correction and empty scattering were applied to data, which were not normalized as samples have the same microtomed thickness. The $\theta$ angle is related to a specific crystalline plane via Bragg's law. The intensity of the peak (100) is not constant with $\varphi^{\prime}$ revealing a preferential crystalline orientation. The integration of the crystalline peak along $2 \theta$ for each direction $\alpha^{\prime}$ gives access to $I\left(\alpha^{\prime}, \varphi^{\prime}\right)$. An example of the measured 2D diffraction pattern is shown in Figure 6. The (100) peak (in red) is clearly visible due to its strong intensity. The large amorphous halo (in green) is also noticeable closer to the beam stop in the center.

Due to the $2 \theta$ angle tilt, the measurement does not give directly access to the diffracting intensity related to the $\mathrm{CD}$. The normal to the diffracting crystalline plane follows a cone that does not contain the CD. Thus, following the previous convention such that $\alpha=0^{\circ}$ corresponds to $\mathrm{CD}$ and $\alpha=90^{\circ}$ to TD, the $\alpha$ angle is obtained by inverting the relation $\cos \alpha=\cos \alpha^{\prime} \cos \theta$, which shows that only the range $\theta \leq \alpha \leq 90^{\circ}$ is accessible. However, relying on the dominance of the second Legendre polynomial for $\cos \varphi$, one can adjust the amplitude of the Hermans factor over this limited angle range, and if needed, one could extrapolate the data up to the $\mathrm{CD}$. 


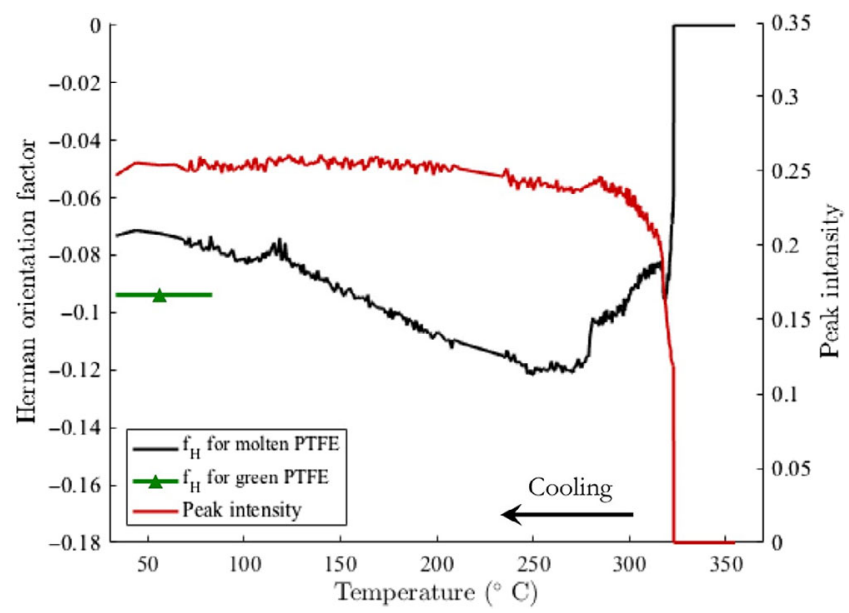

FI G URE 7 Evolution of the Hermans orientation factor of molten PTFE during cooling at $10^{\circ} \mathrm{C} / \mathrm{min}$ compared to its value for green PTFE before melting. The evolution of the crystalline peak intensity (arbitrary unit) is presented to gauge the evolution of the crystallinity [Color figure can be viewed at wileyonlinelibrary.com]

The evolution of $f c$ during cooling at $10^{\circ} \mathrm{C} / \mathrm{min}$ from $370^{\circ} \mathrm{C}$ to ambient temperature is shown in Figure 7. The value before melting, $f c=-0.093$, is indicated. The evolution of the crystalline peak intensity reveals the evolution of the crystallization. It appears that the crystalline orientation sets in at a very early stage of crystallization. This early anisotropy is comparable in magnitude to the one of the green PTFE and even seems to reach higher absolute values, $f c=-0.12$. However, below $270^{\circ} \mathrm{C}$, the anisotropy reduces significantly to reach $f c=-0.073$. The values measured during this experiment are slightly higher than the one measured in the previous experiment both before and after sintering. It could be explained by the fact that the PTFE slices are thinner and therefore some additional shear-induced orientation could result from the microtome cut. The overall evolution is similar for the experiments, and in all cases, sintering is seen to reduce the crystalline texture.

Note that the indicated temperatures are subject to high uncertainties as the measurement was difficult to perform. The thermal gradient from the surface of the hot stage to the outer surface of the PTFE sample can be very high (more than $30^{\circ} \mathrm{C}$ between the two surfaces). And hence the results should be taken as qualitative with respect to the values of temperature, while the revealed trend on the evolution of the crystalline anisotropy during crystallization is believed to be trustworthy.

A few aberrant data points were removed between 210 and $235^{\circ} \mathrm{C}$ (as can be seen by the linear segment in the graphs Figure 7) and not shown for the sake of clarity.

The herein proposed evolution suggests that primary crystallization preserves the crystalline orientation induced by uniaxial compaction. However, the clear trend is a reduction of orientation as temperature is decreased. It could mean that the large amount of secondary crystallization, which produces crystals more isotropically distributed ${ }^{[31]}$ decreases the overall anisotropy. The latter tends to decrease even after the end of crystallization. This could be related to the "glass transition" which has been reported around $120^{\circ} \mathrm{C}^{[32,33]}$ Below $120^{\circ} \mathrm{C}$, the orientation seems stable.

\section{4 | CONCLUSIONS}

A fast evaluation of the orientation of a transversely isotropic semicrystalline polymer can be very informative. Assuming (a) that the polymer crystals are statistically transversely isotropic along the chain direction and (b) that the processing conditions induce a transverse isotropy of the polymer chains, then the Hermans orientation factor can be computed after a mere (potentially coarse) azimuthal X-ray diffraction acquisition. This orientation assessment reduces significantly the required overall scanning time and is robust to measurement noise. The acquisition can even be a single snapshot using a $2 \mathrm{D}$ detector.

This method applied to the case of sintering of uniaxially compacted PTFE showed the crystalline texture of the material induced by compaction. In situ experiments were performed to follow in real time thanks to the above evaluation methodology the evolution of the crystalline orientation during crystallization. It showed that sintering reduces the crystalline texture. The crystalline texture increases in a first stage (primary crystallization) before slowly decreasing along the secondary crystallization stage. The reduction of orientation appears to stop below the glass transition.

\section{ACKNOWLEDGMENTS}

The authors would like to thank Saint-Gobain for sponsoring this work and especially Xavier Brajer, Monika Brodesser, François Creuzet, René Gy, Ansgar Haeger, and Georges Moineau for their implication in the project. The support of the ANRT through the CIFRE PhD grant of Gabriel Guenoun is gratefully acknowledged. The X-ray diffraction experiments were performed on beamline D2AM BM02 at the European Synchrotron Radiation Facility (ESRF), Grenoble, France. We are grateful to Nathalie Boudet and Nils Blanc at the ESRF for providing assistance in using beamline D2AM BM02.

\section{ORCID}

Gabriel Guenoun (1) https://orcid.org/0000-0002-8552-

9821 


\section{REFERENCES}

[1] I. M. Ward Ed., Structure and Properties of Oriented Polymers, Chapman and Hall, London, England 1997.

[2] J. L. White, J. E. Spruiell, Polym. Eng. Sci. 1983, 23, 247.

[3] K. C. Cole, A. Ajji, Chapter 3: Characterization of orientation in solid phase processing of polymers. in Solid Phase Processing of Polymers (Eds: I. M. Ward, P. D. Coates, M. M. Dumoulin), Munich, Hanser 2000, p. 33.

[4] H. Ben, K. C. C. Daly, K. T. Nguyen, B. Sanschagrin, J. Appl. Polym. Sci. 2007, 104, 1319.

[5] P. Hermans, P. Platzek, Kolloid Z 1939, 88, 68.

[6] J. J. Hermans, P. H. Hermans, D. Vermaas, A. Weidinger, Recl. Trav. Chim. Pays-Bas 1945, 65(6), 427.

[7] S. Nomura, H. Kawai, I. Kimura, M. Kagiyama, J. Polym. Sci. A Polym. Phys. 1970, 8, 383.

[8] R. Mendoza, G. Régnier, W. Seiler, J. Lebrun, Polymer 2003, 44(11), 3363.

[9] A. H. Windle, Chapter 1: Measurement of molecular orientation and structure in non-crystalline polymers by wide angle X-ray diffraction. in Developments in Oriented Polymers - 1 (Ed: I. M. Ward), Applied Science Publishers, London, England 1982, p. 1.

[10] G. H. Kim, C.-K. Kang, C. G. Chang, D. W. Ihm, Eur. Polym. J. 1997, 33, 1633.

[11] R. S. Stein, J. Polym. Sci. A 1958, 31(123), 327.

[12] R. S. Stein, J. Polym. Sci. A 1958, 31(123), 335.

[13] Z. W. Wilchinsky, J. Appl. Phys. 1959, 30, 792.

[14] R. A. Sack, J. Polym. Sci. A 1961, 54, 543.

[15] Z. W. Wilchinsky, J. Appl. Phys. 1960, 31, 1969.

[16] Z. W. Wilchinsky, J. Appl. Polym. Sci. 1963, 7, 923.

[17] Y. Wang, M. Cakmak, Polymer 2001, 42(9), 4233.

[18] Z. W. Wilchinsky, J. Polym. Sci. A Polym. Phys. 1968, 6, 281.

[19] W. F. Maddams, J. E. Preedy, J. Appl. Polym. Sci. 1978, 22, 2721.
[20] J. L. White, J. E. Spruiell, Polym. Eng. Sci. 1981, 21, 859.

[21] J. Lu, H.-J. Sue, Macromolecules 2001, 34, 2015.

[22] H. Okuyama, T. Kanamoto, R. S. Porter, J. Mater. Sci. 1994, 29, 6485.

[23] R. Endo, T. Kanamoto, J Polym Sci B 2001, 39, 1995.

[24] D. Sawai, D. Watanabe, N. Morooka, H. Kuroki, T. Kanamoto, J Polym Sci B 2006, 44, 3369.

[25] Y. Takagi, J.-C. Lee, S.-I. Yagi, H. Yamane, T. Wano, D. Kitagawa, A. El Salmawy, Polymer 2011, 52, 4099.

[26] G. Guenoun, J. Y. Faou, G. Régnier, N. Schmitt, S. Roux, Polym. Test. 2019, 75, 99.

[27] M. H. J. K. Li, W. H. de Jeu, Macromolecules 2003, 36, 1626.

[28] T. Ozawa, Bull. Chem. Soc. Jpn. 1984, 57, 952.

[29] R. Kolb, C. Wutz, N. Stribeck, G. von Krosigk, C. Riekel, Polymer 2001, 42, 5257.

[30] M. J. Jenkins, K. L. Harrison, Polym. Adv. Technol. 2006, $17,474$.

[31] G. Guenoun, J. Y. Faou, G. Régnier, N. Schmitt, S. Roux, Polymer 2020, 193, 122333.

[32] G. Dlubek, A. Sen Gupta, J. Pionteck, R. Häßler, R. KrauseRehberg, H. Kaspar, K. Lochhaas, Polymer 2005, 46, 6075.

[33] G. Calleja, A. Jourdan, B. Ameduri, J.-P. Habas, Eur. Polym. J. 2013, 49, 2214. 